\title{
Characterization and comparative profiling of ovarian microRNAs during ovine anestrus and the breeding season
}

Ran $\mathrm{Di}^{1+}$, Jianning He ${ }^{1 \dagger}$, Shuhui Song ${ }^{2+}$, Dongmei $\operatorname{Tian}^{2 \dagger}$, Qiuyue Liu' ${ }^{1}$ Xiaojun Liang ${ }^{3}$, Qing Ma ${ }^{3}$, Min Sun ${ }^{2}$, Jiandong Wang ${ }^{3}$, Wenming Zhao ${ }^{2}$, Guiling Cao ${ }^{1}$, Jinxin Wang ${ }^{1}$, Zhimin Yang ${ }^{1}$, Ying Ge ${ }^{1}$ and Mingxing Chu ${ }^{1 *}$

\begin{abstract}
Background: Seasonal estrus is a critical limiting factor of animal fecundity, and it involves changes in both ovarian biology and hormone secretion in different seasons. Previous studies indicate that two classes of small RNAs (miRNAs and piRNAs) play important regulatory roles in ovarian biology. To understand the roles of small RNA-mediated post-transcriptional regulation in ovine seasonal estrus, the variation in expression patterns of ovarian small RNAs during anestrus and the breeding season were analyzed using Solexa sequencing technology. In addition, reproductive hormone levels were determined during ovine anestrus and the breeding season.

Results: A total of 483 miRNAs (including 97 known, 369 conserved and 17 predicated novel miRNAs), which belong to 183 different miRNA families, were identified in ovaries of Tan sheep and Small Tail Han (STH) sheep. Compared with the three stages of the breeding season, 25 shared significantly differentially expressed (including 19 up- and six down-regulated) miRNAs were identified in ovine anestrus. KEGG Pathway analysis revealed that the target genes for some of the differentially expressed miRNAs were involved in reproductive hormone related pathways (e.g. steroid biosynthesis, androgen and estrogen metabolism and GnRH signaling pathway) as well as follicular/luteal development related pathways. Moreover, the expression of the differentially expressed miRNAs and most of their target genes were negatively correlated in the above pathways. Furthermore, the levels of estrogen, progesterone and LH in ovine anestrus were significantly lower than those in the breeding season. Combining the results of pathway enrichment analysis, expression of target genes and hormone measurement, we suggest that these differentially expressed miRNAs in anestrus might participate in attenuation of ovarian activity by regulating the above pathways. Besides miRNAs, a large and unexpectedly diverse set of piRNAs were also identified.
\end{abstract}

Conclusions: The miRNA profiles of ovine ovaries in anestrus were presented for the first time. The identification and characterization of miRNAs that are differentially expressed between ovine anestrus and the breeding season will help understanding of the role of miRNAs in the regulation of seasonal estrus, and provides candidates for determining miRNAs which could be potentially used to regulate ovine seasonal estrus.

Keywords: Sheep, Seasonal estrus, Anestrus, Ovary, miRNA, piRNA

\footnotetext{
* Correspondence: mxchu@263.net

${ }^{\dagger}$ Equal contributors

${ }^{1}$ Key Laboratory of Farm Animal Genetic Resources and Germplasm Innovation

of Ministry of Agriculture, Institute of Animal Science, Chinese Academy of

Agricultural Sciences, No. 2, Yuanmingyuan West Rd, Beijing, China

Full list of author information is available at the end of the article
}

C Biomed Central (c) 2014 Di et al.; licensee BioMed Central Ltd. This is an Open Access article distributed under the terms of the Creative Commons Attribution License (http://creativecommons.org/licenses/by/4.0), which permits unrestricted use, distribution, and reproduction in any medium, provided the original work is properly credited. The Creative Commons Public Domain Dedication waiver (http://creativecommons.org/publicdomain/zero/1.0/) applies to the data made available in this article unless otherwise stated. 


\section{Background}

Many kinds of animal, such as sheep, horse, bird and fish, are seasonal breeders. Seasonal breeding is a critical limiting factor of animal fecundity. Most sheep in Northern China are estrous in the short-day season and anestrus in the long-day season [1]. Therefore, ewes cannot mate during anestrus and no lambs can be born for several months. Thus, for the mutton industry, seasonal estrus has been a bottleneck limiting efficient lamb meat supply throughout the year.

MicroRNAs (miRNAs) belong to a large family of endogenous non-coding RNAs and have emerged as important post-transcriptional regulators. Many miRNAs have been identified in hircine ovary [2,3], mammary gland [4], testis [5], skin [6], hair [7], or ovine muscle [8]. Recently, genome-wide changes of miRNAs associated with follicular-luteal transition were identified in ovine ovary [9]. Previous studies in mice and pig indicated that miRNAs play critical roles in almost all ovarian biological processes [10-12]. For sheep, the obvious difference of ovarian biology exists between ovine anestrus and the breeding season. However the roles of miRNAs in the regulation of ovine seasonal estrus are unknown. Moreover, to date, only 153 (105 precursors) ovine miRNAs have been identified and listed in the miRBase database (Release 20). The characteristics of miRNA profiles in the ovine anestrus ovary are still unclear.

PIWI-interacting RNAs (piRNAs) are a recently discovered class of small non-coding RNA with a typical length between $23 \mathrm{nt}$ and $32 \mathrm{nt}$ [13-15]. They are typically clustered in the genome in a mono-directional or bidirectional manner. They are highly expressed in sperm cells and have also been identified in oocytes and ovaries of Drosophila and mammals [16-19]. The piRNA pathway is essential in early oogenesis and in transposon silencing in follicle cells $[16,20]$. However, there has been no description of piRNAs in the ewe ovary until now.

To understand the roles of sRNA-mediated posttranscriptional regulation in ovine seasonal estrus, it is necessary to identify the differentially expressed small RNAs in ovine ovaries during anestrus and the breeding season. In Northern China, the Small Tail Han (STH) sheep is a famous local breed because of its year-round estrus and prolificacy. Tan sheep is another popular breed in Northern China because of its delicious meat and excellent lamb skin; however, it has a long anestrus stage usually from April to July. Therefore, the two breeds were considered ideal models for this study.

In this study, the expression profiles of small RNAs in ewe ovaries were compared between anestrus (A) and three distinct stages (luteal phase-L, proestrus-P and estrus-E) during the breeding season using Solexa sequencing technology. KEGG Pathway analyses were then implemented on target genes of the differentially expressed miRNAs. The correlation between the expressions of miRNA and their target genes in reproduction related pathways were analyzed. Since seasonal estrus involves changes in hormone secretion in different seasons, the reproductive hormone levels were also determined in Tan and STH sheep during anestrus and the breeding season. Collectively, these esults will help us to understand the role of miRNAs in the regulation of seasonal estrus.

\section{Results}

Dynamic changes of ovine hormone levels between anestrus and the breeding season

Hormone fluctuation is a characteristic of reproductive status transition. The reproductive hormones levels in Tan and STH sheep were detected in anestrus and at different stages of the breeding season (Figure 1). Briefly, the levels of estrogen, progesterone and luteinizing hormone (LH) in anestrus were significantly lower compared to those in the breeding season $(P<0.01)$. Specifically, the secretion patterns of the four hormones were different. A high concentration of estrogen can promote estrus while a low level inhibits estrus. Our results showed that its secretion gradually increased from anestrus to the luteal phase, proestrus and estrus. Progesterone is a hormone secreted mainly by the corpus luteum, and it showed the lowest level in anestrus and the highest level in the luteal phase. Follicle-stimulating hormone (FSH) and LH are two important endocrine regulators of follicular growth, development and ovulation. Our results demonstrated that their concentrations in anestrus were significantly lower $(P<0.01)$ than those in proestrus and estrus. The hormone results also confirmed that our samples used to perform RNA-seq were collected from the right stages.

\section{Overview of Solexa-sequencing of ovine ovary small RNAs} To understand the expression pattern of ovarian small RNAs in anestrus and the three stages during the breeding season, eight sRNA libraries representing the different stages were constructed from eight adult Tan and STH sheep ovary samples [including: anestrous Tan ewes in spring (TSA), Tan ewes in luteal phase in autumn (TAL), proestrous Tan ewes in autumn (TAP), estrous Tan ewes in autumn (TAE); STH ewes in luteal phase in spring (HSL), proestrous STH ewes in spring (HSP), estrous STH ewes in spring (HSE), estrous STH ewes in autumn (HAE)]. The small RNA libraries were subsequently sequenced and a total of $156,694,170$ raw reads were obtained. After removing adapters and discarding sequences shorter than $17 \mathrm{nt}$ or longer than $35 \mathrm{nt}, 128,389,756$ clean reads remained (Additional file 1) for further analysis. For all samples, $65-89 \%$ of the clean distinct tags (unique sRNAs) for each library were retained by clustering the same reads and aligning to the sheep genome (NCBI, Oarv3.1). For example, in 9,062,723 clean reads of the 


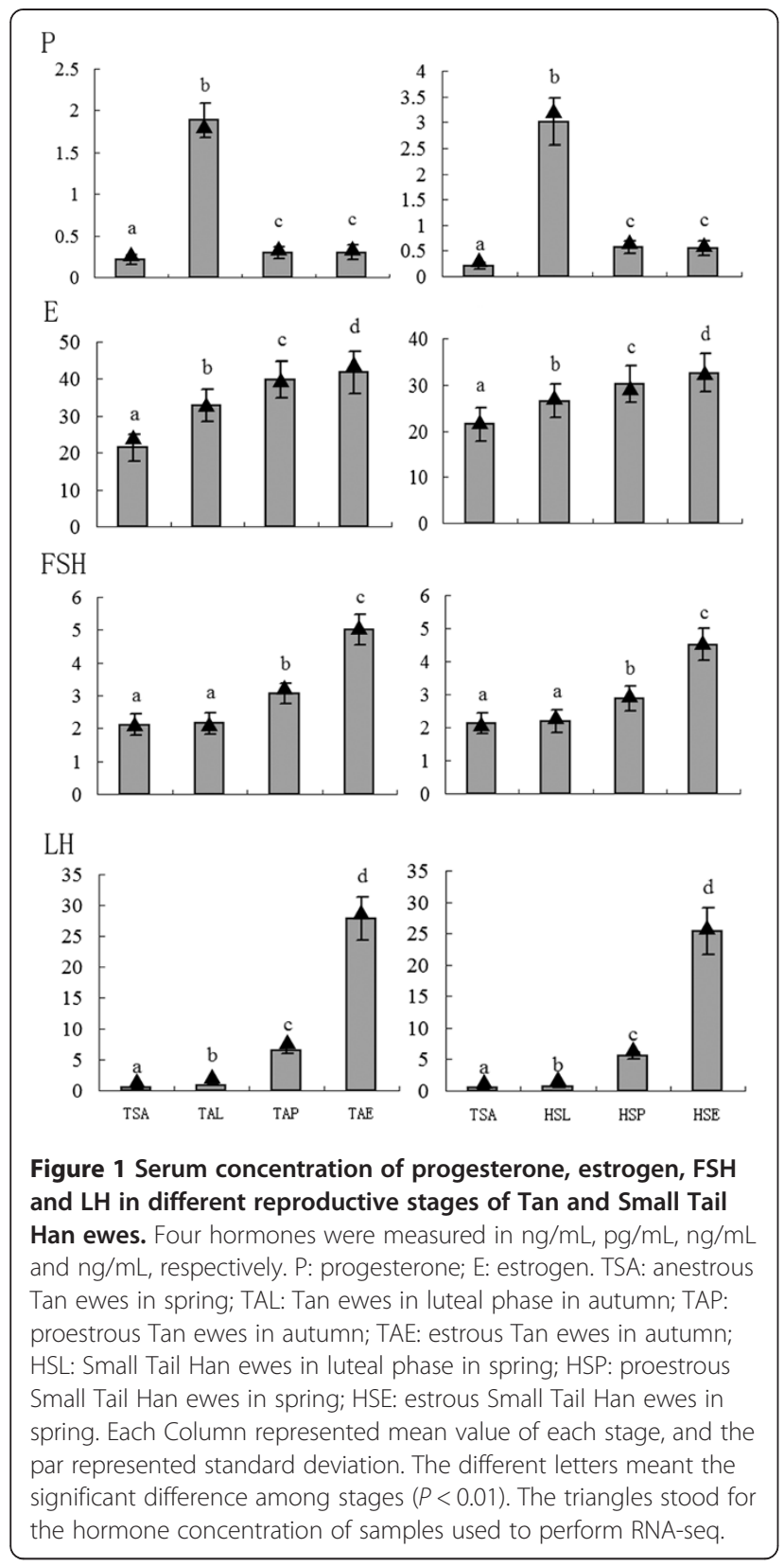

TSA sample, 7,049,153 reads representing $77.78 \%$ of total clean reads were aligned to the sheep genome sequence (Additional file 1). In STH sheep, the mapped reads demonstrated a single-peaked length distribution with a peak at $22 \mathrm{nt}$ (Figure 2A) and in Tan sheep, a length distribution with three peaks at $22 \mathrm{nt}, 29 \mathrm{nt}$ and $32 \mathrm{nt}$ (Figure 2B). The 22 nt length class was consistent with the common size of miRNAs, whereas the $29 \mathrm{nt}$ and $32 \mathrm{nt}$ classes might represent piRNAs. To further assess the efficiency of Solexa sequencing for miRNA detection, all of the mapped clean reads were annotated and classified by aligning against non-coding RNAs (ncRNAs) in the Rfam database and miRbase 20.0. For all samples except HAE and TAE, most sRNA sequences were clustered into miRNA sequences (Figure $2 \mathrm{C}, \mathrm{D}$ ). In addition to the highly enriched miRNA sequences, there were also lowly expressed but much more diverse sequences of other ncRNAs, such as rRNAs, tRNAs, mRNAs, snoRNAs and other sRNAs in the Rfam database (Figure 2C, D).

\section{Expression analysis of miRNAs and miRNA families in ovine ovary}

A total of 483 miRNAs were identified in ovine ovary, including 97 known miRNAs, 369 conserved miRNAs and 17 predicated novel miRNAs (Figure 3A). All these miRNAs were classified into 183 miRNA families based on an established miRNA family system (the miRBase family organization). The size of miRNA families varied from 1 to 21 (Figure 3B). Specifically, 123 (67\%) miRNA families had a single member, $33(18 \%)$ had two members, and the mir-154 and mir-2284 families had the most members $(\mathrm{N}=21)$. It is noteworthy that different members in the same miRNA family might display drastically different expression levels. For example, the expression abundance of let- 7 family members varied from 0 to 344,318 reads. The 20 most highly expressed miRNA families were summarized for each sample (Figure 3C). The mir-10 (MIPF0000033), mir-143 (MIPF0000094), let-7 (MIPF0000002) and mir-26 (MIPF0000043) families were found to be preferentially expressed in ovine ovary. In addition to the above four families, the mir319 (MIPF0001104) family was highly expressed in the TSA sample. The sum of miRNA abundance of the top 20 miRNA families represented more than $85 \%$ of total sRNAs in most samples, while this percent only reached $19 \%$ in the TSA sample.

\section{Expression profile of ovarian miRNAs in ovine anestrus}

In this study, the whole expression profile of ovarian miRNAs in ovine anestrus was determined for the first time. In total, 202 expressed miRNAs, including 63 known, 136 conserved and three novel predicated miRNAs were identified (Figure 4A). Compared to the other three stages, the number of expressed miRNAs is the smallest and the total abundance of the top 20 miRNA families is approximately $19 \%$ of all expressed sRNAs in anestrus stage sample, which suggests that novel miRNAs were highly expressed in anestrus. Therefore, miRNAs in each sample were also ranked based on their level of expression (Figure 4B). In the 33 most highly expressed miRNAs, miR-n-142 (homology ID: aca-miR-5441), a newly identified conserved miRNA, was the most abundant miRNA in anestrus which accounted for $82 \%$ of the total expressed miRNAs. KEGG pathway analysis showed that the target genes of miR-n-142 were predominantly enriched in oxidative phosphorylation, glycerolipid metabolism and phosphatidylinositol signaling pathways. miR-n-789 


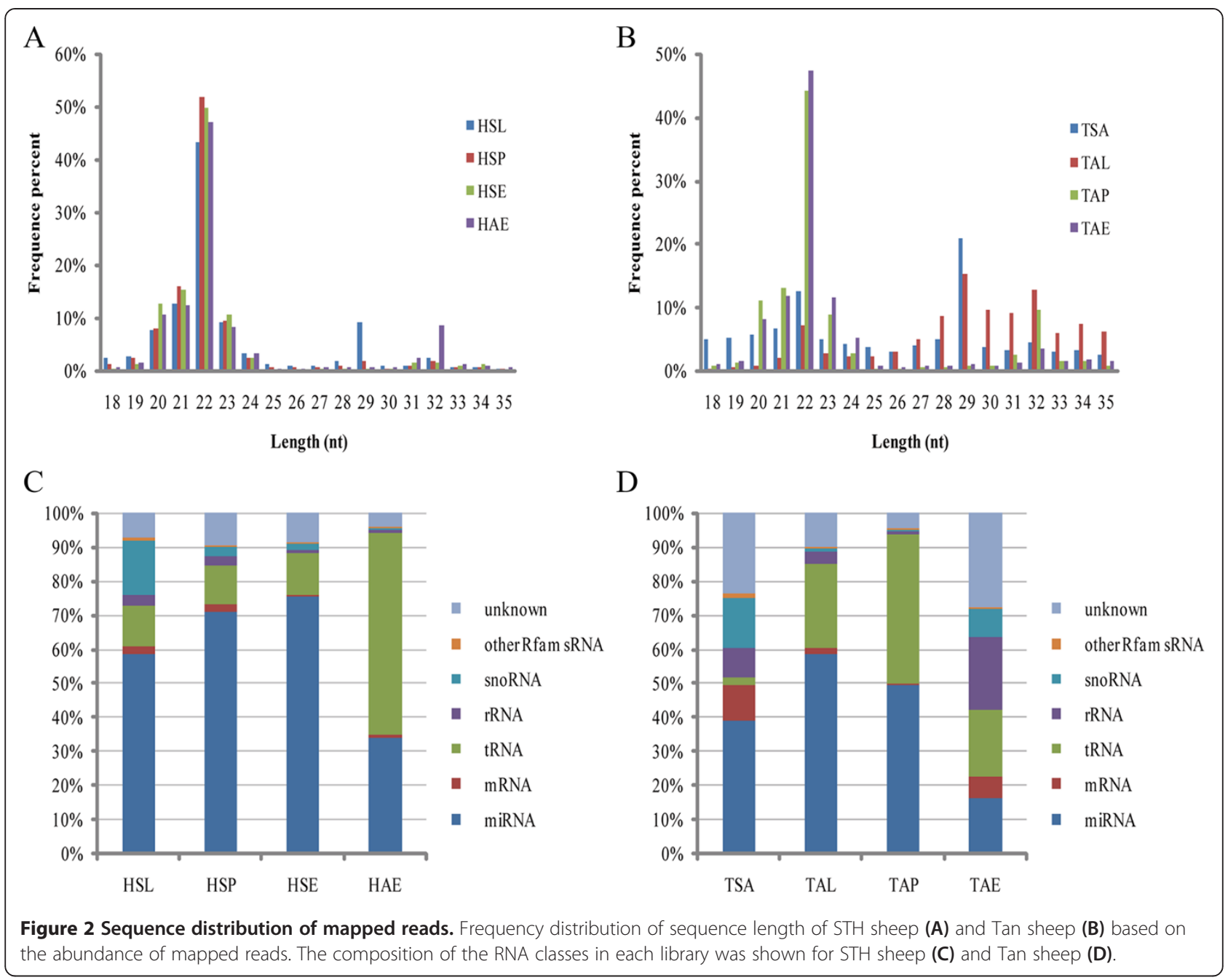

(Homology ID: ptc-miR319i) is another remarkably highly expressed miRNA in anestrus, and its target genes were enriched in circadian rhythm, notch signaling and the GnRH signaling pathway. The other highly expressed miRNAs, such as oar-let-7a, oar-miR-21 and oar-miR-125b have been reported to be highly expressed in ovine ovarian follicles [2]. The newly identified highly expressed miRNAs in anestrus will facilitate understanding of their new roles in seasonal estrus.

\section{Comparative analysis of the differentially expressed miRNAs between anestrus and the other three stages during the breeding season}

The ovarian miRNA expression profile in anestrous Tan sheep was compared with those in the other three stages in both Tan and STH sheep (Figure 5A). In total, 58, 57 and 27 significantly differentially expressed miRNAs were identified between anestrus $v s$. luteal phase, anestrus vs. proestrus and anestrus vs. estrus, respectively.
For these significantly differentially expressed miRNAs, their expression patterns (up- or down-regulation) between anestrus of Tan and the other stages of Tan were basically consistent with those between anestrus of Tan and the other stages of STH sheep (Figure 5B). With the aim of intensively identifying miRNAs associated with anestrus, we finally focused on 25 shared miRNAs that were significantly differentially expressed between anestrus and other three stages (Figure 5C). Of the 25 differentially expressed miRNAs, 19 were significantly up-regulated and 6 were significantly down-regulated in anestrus. The precursor sequences and chromosome locations of the 14 most highly up-regulated miRNAs were predicated and are shown in Table 1. Hairpin structures of six significantly down-regulated miRNAs are shown in Figure 6. To understand the function of these differentially expressed miRNAs, KEGG pathway analysis was performed. Some target genes of these miRNAs were enriched in reproduction related pathways (Table 1 and Figure 6), such as the 
reproductive hormone related pathways (e.g. androgen and estrogen metabolism, steroid biosynthesis and GnRH signaling pathway) as well as follicular (or luteal) development related pathways (e.g. Notch and MAPK signaling pathway and progesterone-mediated oocyte maturation). Simultaneously, the expression levels of predicted target genes of the differentially expressed miRNAs were extracted and analyzed from RNA-sequencing results (unpublished). By correlation analysis, we found that the expression levels of these differentially expressed miRNAs were negatively correlated with most of their target genes in the above pathways (Additional file 2). Finally, a network that is composed of the differentially expressed miRNAs, their target genes and enriched KEGG pathways is shown in Figure 7. Some miRNAs shared the same target genes or reproduction related pathways.

To verify the Solexa sequencing data, five miRNAs (miR-n-13, miR-n-162, miR-n-180, oar-miR-374b and miR-n-17) were randomly selected and qRT-PCR for all reproductive stages was conducted. There was a strong positive correlation $\left(R^{2}=0.975\right)$ between RNA-seq and qRT-PCR data indicating consistency of results. 


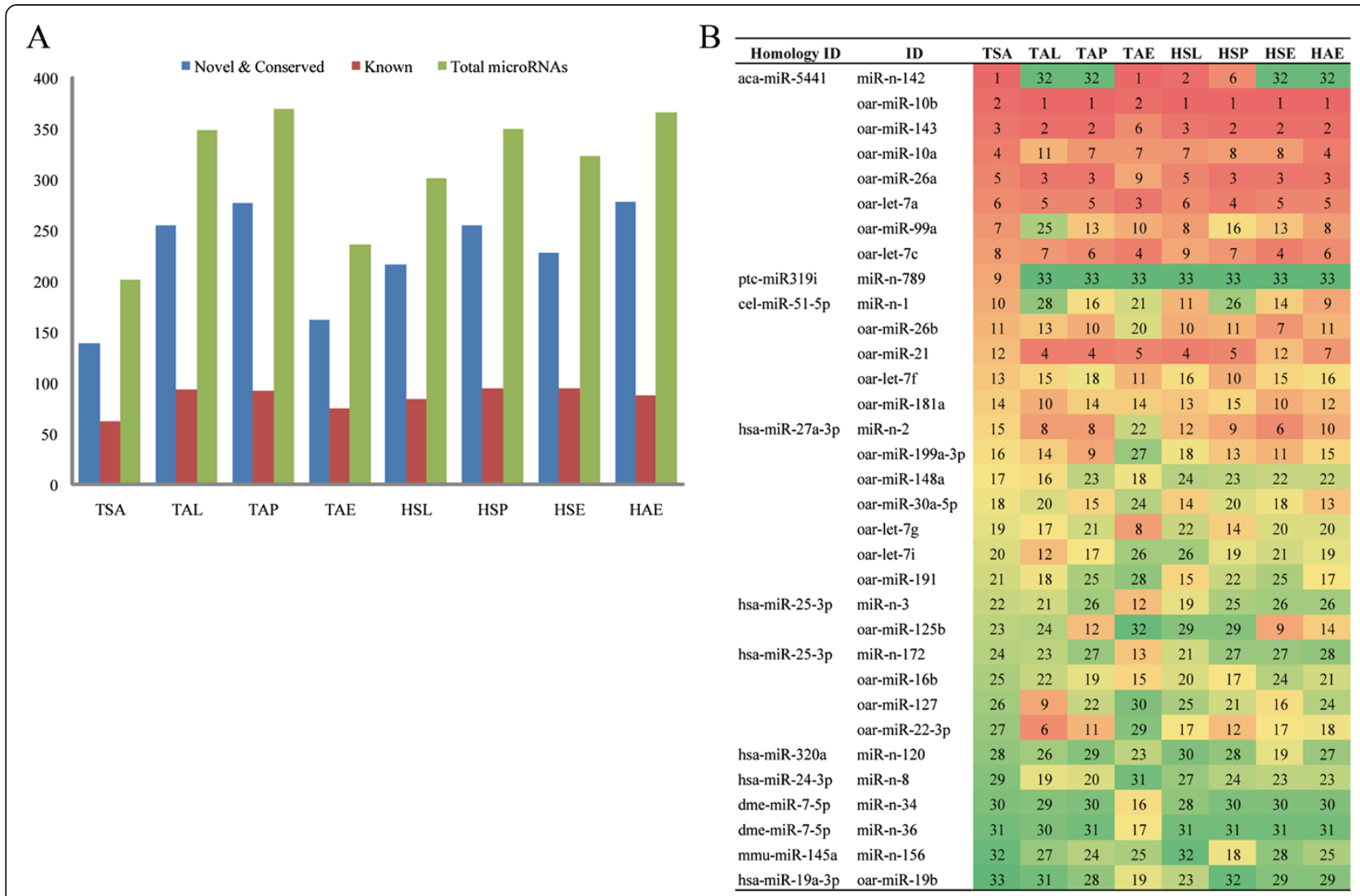

Figure 4 Comparison of the miRNA expression profiles between anestrus and the other three stages. (A) Expressed miRNAs for each sample; (B) The top expressed miRNAs in each sample.

\section{Predication of piRNAs from ovine ovary Solexa sequencing data}

piRNA pathways are particularly essential for genome integrity, retrotransposon repression and germ cell development. piRNAs have been identified in $\mathrm{CHO}$ cells and oocytes of mouse [21,22]. In our ovine ovary sequenced libraries, a distinct subset of small RNAs ranging from 24 to $33 \mathrm{nt}$ was detected and they were derived from ewe genomic regions that do not encode miRNAs. These small RNAs were characterized using two methods. By de novo prediction, a total of 166,164 (23,288 piRNAs with read numbers $>5$ ) candidate piRNAs were predicated (Figure 8A). Homology searches against to known piRNAs in piRNABank identified 13,920 homologous piRNAs (Figure 8A). Of all of the predicated piRNAs, 3,740 sequences were also identified by homology search. These piRNAs displayed a highly uneven distribution among chromosomes and were enriched on chromosomes 11, 1, 2, 3 and X (Figure 8B). The predicated piRNAs came from 1,230 piRNA cluster loci. Each cluster locus contained 36-10,858 piRNAs (an average of 201 piRNAs) and spanned from 1,251 to 177,943 bp (an average of 15,951 bp). According to the definition of piRNA cluster types described by proTRAC, 1,002 clusters were mono-directional while 198 clusters were bi-directional and distributed on two strands, and only 30 clusters had no directional orientation.

\section{Discussion}

Tan and STH sheep are two well known breeds in Northern China, and they are seasonal and year-round breeders, respectively. To understand the roles of sRNAmediated post-transcriptional regulation in ovine seasonal estrus, we compared the small RNA profiles of ovarian tissues between anestrus of Tan sheep and three stages during the breeding season of both Tan and STH sheep using Solexa sequencing technology.

miRNAs with high expression levels in anestrus and other stages during the breeding season

The information in the ovine miRNA database has been enriched by this study and this is the first report of miRNA expression profiles of anestrous ovine ovary tissues. Some of the abundant miRNAs in the anestrous ovary of Tan sheep, including miR-143, miR-26a, let-7 and miR-21, were also reported to be highly abundant in ovaries of human, cow [23-25], pig [26], adult and neonatal mouse $[27,28]$ and sheep [2,3]. Moreover, according 


\begin{tabular}{|c|c|c|c|c|c|c|c|}
\hline & \multicolumn{2}{|c|}{ Luteal phase } & \multicolumn{2}{|c|}{ Proestrus } & \multicolumn{3}{|c|}{ Estrus } \\
\hline & TAL & HSL & TAP & HSP & TAE & HAE & HSE \\
\hline Anestrus (TSA) & 110 & 93 & 98 & 93 & 103 & 53 & 73 \\
\hline Co-SDE miRNAs & \multicolumn{2}{|c|}{58} & \multicolumn{2}{|c|}{57} & & 27 & \\
\hline
\end{tabular}

B

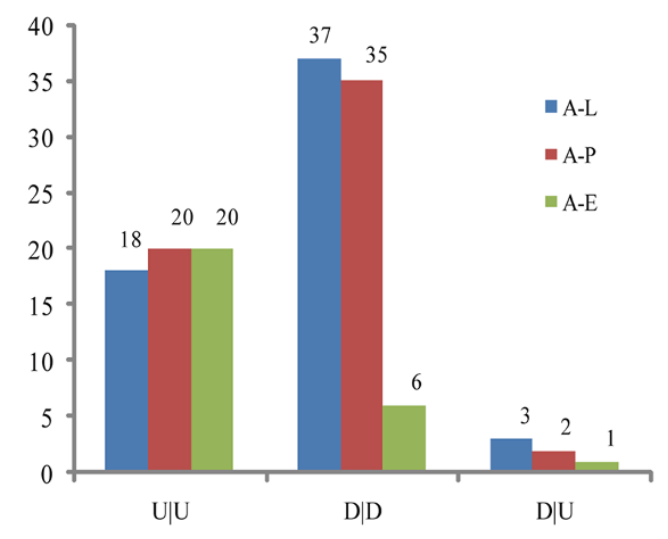

$\mathrm{C}$

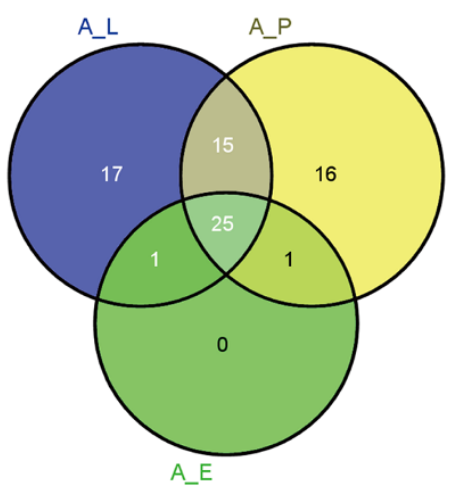

Figure 5 Differentially expressed miRNAs between anestrus and the other stages during estrus cycle. (A) The number of differentially expressed miRNAs. (B) The number of down-regulated miRNAs (indicates in D) and up-regulated (indicates in $\mathrm{U}$ ) genes in anestrus when compared to the other three stages during estrus cycle. A-L: anestrus vs. luteal phase; A-P: anestrus vs. proestrus; A-E: anestrus vs. estrus. "U/U" and "D/D": the expression patterns (up-regulation or down-regulation) of miRNAs between anestrus of Tan and the other stages of Tan were basically consistent with those between anestrus of Tan and the other stages of STH sheep; "D/U" means differentially expressed miRNAs are inconsistent between above two comparisons. (C) Venn diagram showed the 25 overlapped differentially expressed miRNAs between anestrus and the other three stages.

to our results, these miRNAs were also highly expressed in ovine ovaries of different stages during the breeding season. This conserved high level of expression across mammalian species and reproductive stages suggests their important roles in mammalian ovarian function. In previous studies, miR-21 was identified to be able to promote follicular cell survival during ovulation [29], miR-143 was critical for the formation of mouse primordial follicles [30] and let-7b was shown to be necessary for normal development of the corpus luteum [10]. Additionally, some KEGG pathways, in which the target genes of these miRNA were enriched, are important for ovarian function. For instance, miR-143 was significantly enriched in the GnRH signaling and progesterone-mediated oocyte maturation pathways, which are crucial for ovarian activity by influencing endocrine function and follicular development. In addition, the apoptosis, Wnt signaling, and C21-Steroid hormone metabolism pathways, which contain target genes of the let-7 family, are important for granulosa cell proliferation, follicular growth and development, and luteinic formation and regression. More importantly, miR-n-142 (homology ID: aca-miR-5441), a novel predicated conserved miRNA, was the most highly expressed miRNA in anestrus (accounting for $82 \%$ of total miRNA expression). Its target genes were mainly involved in oxidative phosphorylation, glycerolipid metabolism, and phosphatidylinositol signaling pathways. Further research is needed to reveal its potential role in reproduction.

\section{Differentially expressed miRNAs between anestrus and the other three stages in the breeding season}

Besides the miRNAs with high levels of expression in anestrus, 25 miRNAs that were differentially expressed between anestrus and the other three stages were also identified, and most of them were novel. Of these, only the function of miR-n-145 had been studied and it is mainly involved in the regulation of proliferation of granulosa cells. Yan et al. reported that miR-n-145 mediated attenuation of activin-induced proliferation of mouse granulosa cells by targeting both activin receptor $1 \mathrm{~B}$ and cyclin D2 [31]. When cultured bovine granulosa cells were treated with forskolin (an adenylate cyclase agonist) to promote luteinization, a decrease in the levels of miR-n145 was observed during the follicular-luteal transition [2]. These results implied that miR-n-145 might mediate inhibition of granulosa cell proliferation during the follicular stage. During the ovine breeding season, the ovary is in the estrus cycle, including the follicular stage and luteal phase, so it is likely that the high level of miR-n-145 expression was involved in attenuating the proliferation of ovine 
Table 1 Precursor sequences, genome locations and enriched KEGG pathways of significantly up-regulated miRNAs in anestrus

\begin{tabular}{|c|c|c|c|c|c|c|}
\hline miRNA & Chromosome & Start & End & Strand & miRNA precursor sequence & Enriched KEGG pathways \\
\hline miR-n-783 & chr26 & 38599891 & 38599941 & + & ccaccgggcuccucugaccacgggacuucccagagaagacugcagugggu & $\begin{array}{l}\text { MAPK signaling pathway; GnRH signaling } \\
\text { pathway; Oxidative phosphorylation }\end{array}$ \\
\hline miR-n-786 & chr21 & 8878698 & 8878742 & + & uugguguaugugcuuggcugagaagccaauggggcgaagcuacc & $\begin{array}{l}\text { Androgen and estrogen metabolism; } \\
\text { Fatty acid metabolism }\end{array}$ \\
\hline miR-n-784 & chr7 & 84685572 & 84685648 & - & $\begin{array}{l}\text { ccacggacuguacaguccuuggggcugcaaagagucggacaggac } \\
\text { ugagcucuuucaggaaugacaguccccaguc }\end{array}$ & Basal transcription factors; Fatty acid biosynthesis \\
\hline miR-n-787 & chr16 & 496440 & 496499 & + & $\begin{array}{l}\text { cugggcuccucugucugaggugacaaugugucaaaguccca } \\
\text { gcaagagagaguccacag }\end{array}$ & Notch signaling pathway; Steroid biosynthesis \\
\hline miR-n-444 & chr16 & 39273458 & 39273523 & - & $\begin{array}{l}\text { caguucccucuuccuugccaguggagaugacagugcucuccaag } \\
\text { aagcuggaggggaggacuucc }\end{array}$ & Notch signaling pathway; Apoptosis \\
\hline miR-n-782 & chr2 & 111819172 & 111819244 & + & $\begin{array}{l}\text { cguggaucaaagcaucuauggauuucccucgugucuuucccacgagge } \\
\text { uuuccaacgaggcuuucccacagg }\end{array}$ & Metabolism of xenobiotics by cytochrome P450 \\
\hline miR-n-793 & chr4 & 118592936 & 118593008 & - & $\begin{array}{l}\text { cguggaucaaagcaucuaugguuuucccucgugucuuucccacgagge } \\
\text { uuucccacgaggcuuucccacagg }\end{array}$ & Metabolism of xenobiotics by cytochrome P450 \\
\hline miR-n-792 & chr16 & 38836730 & 38836775 & - & uccuunguaaacaucuggaaagccaagcuguaaacucugaggauc & - \\
\hline miR-n-790 & chr24 & 5171923 & 5171991 & - & $\begin{array}{l}\text { ccauggcuugguucaauacucuuaaguuacuccucaacccaugg } \\
\text { aagacuaggggacuguuauguguc }\end{array}$ & $\begin{array}{l}\text { Cytokine-cytokine receptor interaction; } \\
\text { MAPK signaling pathway }\end{array}$ \\
\hline miR-n-785 & chr3 & 142054999 & 142055066 & - & $\begin{array}{l}\text { gcaunaugguuacauuguggcugcugcuacagcucuuguuucc } \\
\text { gccaggauggagacuguguuaugg }\end{array}$ & Basal cell carcinoma; PPAR signaling pathway \\
\hline miR-n-788 & chr26 & 232395 & 232460 & + & $\begin{array}{l}\text { cagaguugaggcugggagcuccagggcuaagguccugggac } \\
\text { caggcucugggucucugacuuggg }\end{array}$ & Long-term depression \\
\hline miR-n-791 & chr 5 & 4904837 & 4904905 & + & $\begin{array}{l}\text { ucaggggcaauggccagguggagcugguguggucaguucu } \\
\text { ggggcuccugggecgugugccccuggac }\end{array}$ & $\begin{array}{l}\text { Insulin signaling pathway; GnRH signaling pathway; } \\
\text { MAPK signaling pathway; Notch signaling pathway }\end{array}$ \\
\hline miR-n-795 & chr19 & 52910579 & 52910655 & + & $\begin{array}{l}\text { ccagcauugaggggccaguggaauucuggugcaggcucucu } \\
\text { cccuggcuugccaguggucacuuucuugcuguguc }\end{array}$ & MAPK signaling pathway \\
\hline miR-n-794 & chr2 & 175146052 & 175146125 & + & $\begin{array}{l}\text { caaugagggacuggaccaguuggauucugggguaagaaugc } \\
\text { ucuggguggaaguacuagcuagaacccaggcu }\end{array}$ & - \\
\hline
\end{tabular}




\begin{tabular}{|c|c|c|c|}
\hline miRNA name & Homology ID & Hirpin structure & Enriched KE GG pathways \\
\hline oar-miR-655-3p & hsa-miR-655-3p & & Nucleotide excision repair \\
\hline oar-miR-200a-3p & mmu-miR-141-3p & & Progesterone-mediated oocyte maturation \\
\hline oar-miR-543-3p & mmu-miR-543-3p & & Fatty acid biosynthesis; RNA degradation \\
\hline $\operatorname{miR}-\mathrm{n}-77$ & mmu-miR-330-3p & & $\begin{array}{l}\text { Insulin signaling pathway; } \mathrm{GnRH} \text { signaling } \\
\text { pathway; MAPK signaling pathway }\end{array}$ \\
\hline $\operatorname{miR}-\mathrm{n}-24$ & bta-miR-3432 & & Prion diseases \\
\hline $\operatorname{miR}-\mathrm{n}-145$ & mo-miR-324-5p & & $\begin{array}{l}\text { Cardiac muscle contraction; Cyanoamino } \\
\text { acid metabolism }\end{array}$ \\
\hline
\end{tabular}

Figure 6 Hirpin structure and enriched KEGG pathways of significantly down-regulated miRNAs in anestrus.

granulosa cells. However, during anestrus the physiological activity of the ovary was relatively reduced and the follicular stage does not exist. Thus the proliferation of granulosa cells is significantly attenuated. Therefore, in our study, the low level of miR-n-145 expression observed in anestrus is reasonable.

\section{Reproduction-related pathways in which differentially expressed miRNAs were enriched}

The KEGG pathway analysis indicated that some target genes of the differentially expressed miRNAs were enriched in reproduction-related pathways (Figure 7). First, some pathways were crucial for the secretion of gonadal hormones, and there were some successive relationships among the pathways. Specifically, both miR-n-787 and miR-n-786 were significantly enriched in estrogen-related pathways (Androgen and estrogen metabolism pathway and Steroid biosynthesis pathway), and the target genes of miR-n-791 were significantly enriched in the Insulin signaling pathway. As Additional file 3 shows, the Insulin signaling pathway may act on the estrogen-related pathway and ultimately they can regulate the biosynthesis of gonadal hormone (e.g. estrogen and progesterone) together. According to the RNA-sequencing results, miRn-787 expression was negatively correlated with that of $80 \%$ of its target genes in the Steroid biosynthesis pathway; while the expression of miR-n-786 was negatively correlated with that of target genes (NSUN4 and UGT2C1) in the Androgen and estrogen metabolism pathway. Previous studies have indicated that the ewes in anestrus had relatively lower estrogen and progesterone levels compared with in the breeding season. In anestrus, progesterone secreted by the corpus luteum is suppressed because fewer ovulations led to a reduction of the total luteal volume; although it may also result from diminished gonadotropic support (e.g. LH). Our results for hormone determination also indicated that the level of estrogen and progesterone in anestrus of Tan sheep were significantly lower than those in the breeding season of Tan and STH sheep. These results suggest that miR-n-786 and miR-n-787 might play roles in the estrogen-related pathways by negatively regulating their target genes.

Second, the enriched KEGG pathways of differentially expressed miRNAs are important for follicular and luteal development. Targets of both miR-n-787 and miR-n-789 were enriched in the Notch signaling pathway, which is necessary for follicular development [32,33] and vessel formation in the follicle and corpus luteum [34]. Correlation analysis indicated that the expression of miR-n-787 and miR-n-789 was negatively correlated with most of their target genes involved in the Notch signaling pathway. Trombly et al. [33] reported that the suppression of Notch signaling with gamma-secretase inhibitors in the neonatal mouse ovary could decrease primordial follicle formation. Complete blockage of the Notch signaling pathway with compound $\mathrm{E}$ also impairs folliculogenesis and induces disruption of gonadotropin stimulated angiogenesis [32]. Vorontchikhina et al. suggested that Notch signaling plays an important role in angiogenic growth during mouse folliculogenesis and in the corpus luteum [34]. In the breeding season, the vessels are rich in the antral follicle and corpus luteum; however, in anestrus, little vessel formation occurs because of the absence of ovulation and the corpus luteum. Collectively, these results indicate that miR-n-787 and miR-n-789 might play regulatory roles during vessel formation in the follicle and corpus luteum via the Notch 


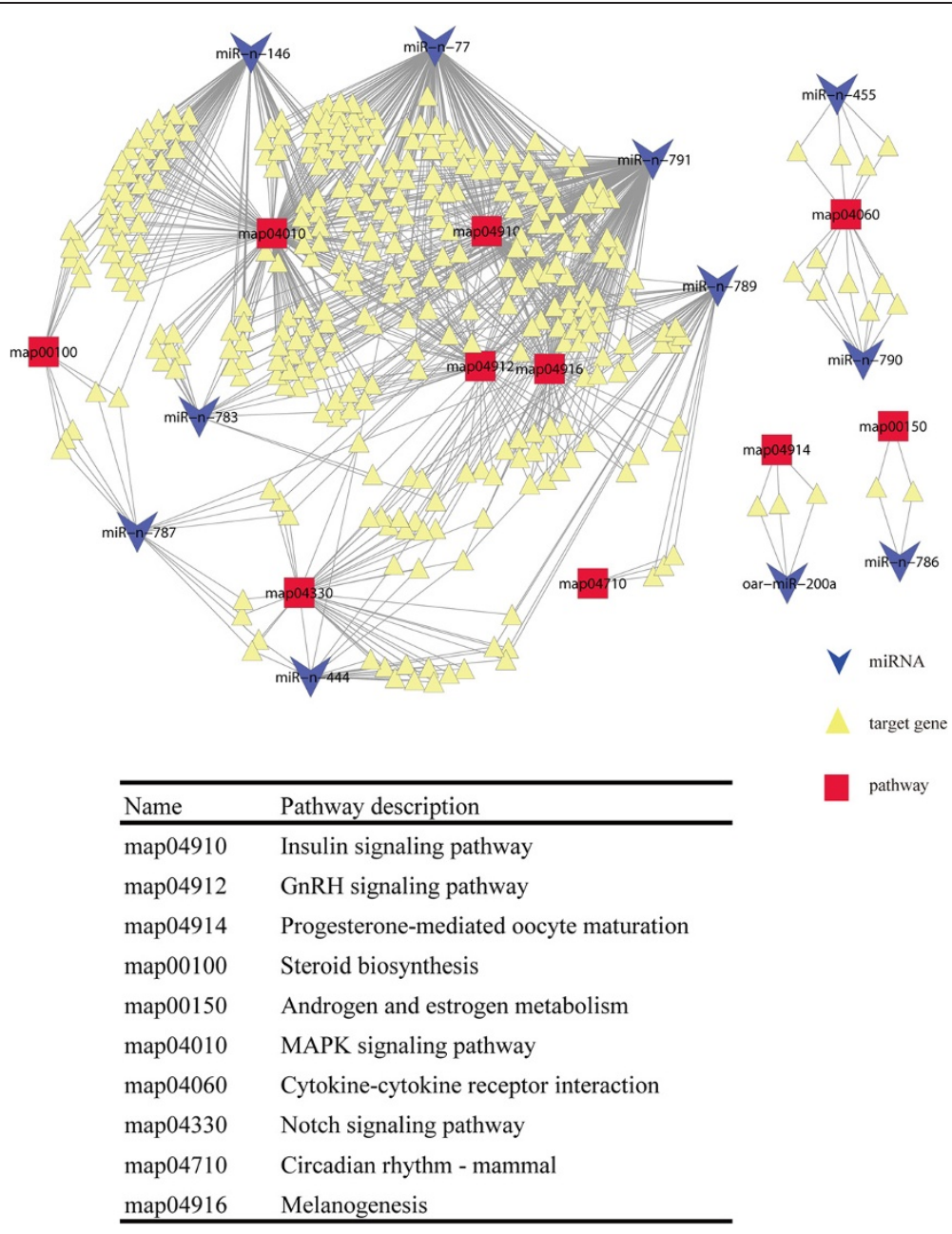

Figure 7 Network consisting of differentially expressed miRNAs, their target genes and enriched KEGG pathways.

A

\begin{tabular}{ll}
\hline Number of piRNAs found by proTRAC & 166164 \\
Number of piRNAs filtered" & 13540 \\
Number of piRNAs found by homology search & 13920 \\
Overlapped piRNAs found by proTRAC and homology search & 3740 \\
Number of clusters & 1230 \\
$\quad$ Number of mono-directional clusters & 1002 \\
Number of bi-directional clusters & 198 \\
Number of non-directional clusters & 30 \\
& \\
Minimum cluster length & $1251 \mathrm{nt}$ \\
Maximum cluster length & $177943 \mathrm{nt}$ \\
Average cluster length & $15951.96 \mathrm{nt}$ \\
Minimum piRNAs/cluster & 36 \\
Maximum piRNAs/cluster & 10858 \\
Average piRNAs/cluster & 201.07 \\
\hline
\end{tabular}

Note: ${ }^{a}$ reads $\mathrm{No}>5$ in at least one sample

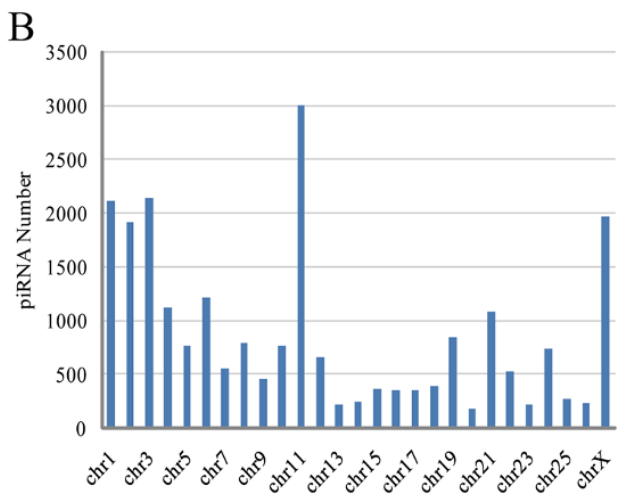

Figure 8 Predicated piRNAs and piRNA clusters. (A) Statistics of predicated piRNAs (B) chromosome distribution of predicated piRNAs with reads numbers $>5$ in at least one sample. 
signaling pathway. Another important pathway for follicular development is the MAPK signaling pathway, and target genes of miR-n-783, miR-n-791 and miR-n-77 were enriched in this pathway. The activation of the MAPK pathway could promote leptin-stimulated oocyte maturation, and conversely the maturation could be blocked when leptin-induced MAPK phosphorylation was suppressed by a specific MAPK activation inhibitor [35]. In addition, the Progesterone-mediated oocyte maturation pathway, in which the target genes of oar-miR-200a-3p were enriched, was related to maturation of the oocyte [36].

Third, it was interesting and unexpected that the target genes of miRNAs including miR-n-783, miR-n-791 and miR-n-77 were simultaneously enriched in the GnRH signaling pathway. In addition, some pathways relating to the GnRH signaling pathway were also involved; for example, the target genes of miR-n-790 were enriched in the Cytokine-cytokine receptor interaction pathway, and target genes of miR-n-783, miR-n-791 and miR-n-77 were synchronously enriched in the MAPK signaling pathway. It is noteworthy that these pathways could connect mutually in a network (Additional file 4) and finally could regulate the secretion of gonadotropins (FSH and $\mathrm{LH}$ ), which are known as the crucial endocrine regulators of follicular growth, development and ovulation. Recent studies in plants, animals and humans show that miRNAs might regulate gene expression in other cells or tissues by migration to target cells or tissues [37-41]. Hence it is likely that, similar to how hormones secreted from the ovary can exert a feedback regulation on gonadotropins (FSH and $\mathrm{LH}$ ) in the hypophysis, these miRNAs might be involved in similar regulation of gonadotropins by targeting genes in the above pathways. It has been reported that a low level of LH is necessary for anestrus $[42,43]$. The hormone levels in Tan and STH ewes also showed that the concentration of LH in Tan anestrus was significantly lower compared to that in the breeding season (Figure 1). Collectively, these differentially expressed miRNAs in ovine anestrus might mediate post-transcriptional regulation on secretion of $\mathrm{LH}$ by the GnRH signaling pathway.

Finally, these findings provide candidates for further experimentation to identify miRNAs which could be potentially used to regulate ovine seasonal estrus.

\section{Predicted piRNAs in the ovine ovary}

piRNAs are a new class of small RNAs that bind to the PIWI argonaute protein family. Large scale sequencing for piRNAs from rat, mouse, fly and human has yielded very high numbers of piRNA sequences, which can be acquired from piRNABank [44]. The piRNA-PIWI complexes have been reported to be involved in post-transcriptional gene silencing of retrotransposons and other genetic elements in germ line cells, particularly during spermatogenesis [45]. They were first discovered in spermatogenic cells
[46] and testicular cells in rat [15]. piRNA also exist in mammalian ovaries [21,47] and the piRNA pathway is essential for early oogenesis [48] and transposon silencing in follicle cells $[16,20]$. Recently, it was found that piRNAs have widespread expression in all macaque tissues [49]. Usually, the length of piRNAs is concentrated at 26-31 nt. In our study, the length distribution of small RNAs showed double-peaks at 21-24 and 24-32 nt, and a considerable percentage (33\%-69\%) of reads were between 24 and 32 nt in length. Because of the lack of identified or previously reported piRNAs in ewe, in the present study, 166,164 candidate piRNAs of 24-33 nt were first identified using de novo prediction and homology search methods, and they were classified into 1,251 clusters. The number of piRNAs was comparable to the number of piRNAs previously found in other organisms listed in the piRNABank database. In our study the piRNAs in the ovine ovary displayed a highly uneven distribution among chromosomes and were enriched on chromosome 11 and X. This characteristic has also been reported in porcine testes [50]. Widespread piRNA expression across all reproductive stages hinted at their important functions in ovarian activity.

\section{Conclusions}

For the first time, the miRNA profiles of the ovine ovary in anestrus are presented. In total, 483 miRNAs were identified in the ovine ovary. The expression patterns of the miRNAs varied between different reproductive stages. Compared with the three stages of the breeding season, 25 shared significantly differentially expressed (including 19 up- and six down-regulated) miRNAs were identified in ovine anestrus. Combining the results of pathway enrichment analysis, expression of target genes and hormone measurement, these differentially expressed miRNAs in anestrus are indicated to participate in attenuation of ovarian activity by regulating the pathways relating to hormone secretion and follicular development. In addition, a large and unexpectedly diverse set of piRNAs sequences were identified in the ewe ovary. Our findings will help understanding of the role of miRNAs in the regulation of seasonal estrus, and provides candidates for identifying miRNAs which could potentially be used to regulate ovine seasonal estrus.

\section{Methods}

\section{Experimental animal tissue collection and hormone determination}

All procedures involving animals were approved by the Animal Care and Use Committee of Chinese Academy of Agricultural Sciences, Beijing, China and the Animal Care and Use Committee of Ningxia Academy of Agriculture and Forestry Sciences, Yinchuan, Ningxia, China. 
Tan sheep and STH sheep were selected from respective breed conservation farms and housed in the same farm in Ningxia Autonomous Region, China. The three years-old, clinically normal and non-pregnant Tan and STH ewes were examined daily for estrous activity with a teaser ram during four seasons. The date of estrous cycle and duration of estrus were recorded and their blood was collected daily for measurement of serum hormone concentrations. Estradiol, FSH and LH were measured by radioimmunoassay methods which have been described in detail [51-53]. Progesterone was measured using a radioimmunoassay method described previously [54] as validated for sheep [55]. Estrus was judged according to the obvious estrous signs in response to the teaser ram. Anestrus was a period without obvious estrous signs during more than 36 days (the time of two estrous cycles). Ewes in luteal phase and proestrus were determined according to records of three consecutive estrous cycles and the method of characterizing particular stages of the estrous cycle by plasma progesterone and LH concentrations [56]. Finally, eight ewes standing for different reproductive stages were selected randomly and killed for ovary collection. All ovary samples were immediately snap-frozen in liquid nitrogen for total RNA extraction.

Reproductive hormone (estrogen, progesterone, FSH and $\mathrm{LH}$ ) levels in different reproductive stages were also analyzed with the above described radioimmunoassay methods in five Tan and five STH 3 year-old ewes. In anestrus, the blood was collected three times a day for 18 days. During an integral estrus cycle (about 18 days) in the breeding season, for both Tan and STH ewes, blood was collected three times a day in luteal stage and proestrus, and once every two hours in estrus. For each hormone, the mean concentration in each stage was calculated and the difference among stages was analyzed using the F-test program of SAS 8.0.

\section{RNA extraction, mRNA and small RNA sequencing}

Total RNA was extracted from ovary tissue at different stages using TRIzol (Invitrogen Carlsbad, CA, USA) according to the manufacturer's instruction.

Low-molecular weight RNAs (<40 nucleotides long) were isolated from total RNA using a FlashPAGE fractionator (Ambion, Life Technologies, Paisley, UK). miRNA libraries were constructed using the Illumina TruSeq Small RNA Sample Preparation kit, in which miRNAs were ligated to two adapters (5' Adapter: 5'-GUUCAGAGUUC UACAGUCCGACGAUC-3'; 3' Adapter: 5'-TGGAATT CTCGGGTGCCAAGG-3') and amplified by RT-PCR, the amplification products (140-160 bases, including the small RNA and adapter sequences) were further purified on a $15 \%$ polyacrylamide TBE gel and sequenced with an Illumina Hiseq 2000 system. The mRNA libraries for different reproductive stages were generated using Illumina Truseq RNA Sample Preparation Kits. The required fragments were enriched by PCR amplification and purified using a Qiagen MiniElute PCR Purification Kit. The library products were sequenced with an Illumina HiSeq 2000 system. The library construction and sequencing of miRNAs and mRNAs was performed at Beijing Institute of Genomics, Chinese Academy of Sciences (Beijing, China).

\section{Bioinformatic analysis of sequenced small RNAs}

All sequenced raw fastq reads from each small RNA library were used for miRNA analysis. First, the 3' primer adaptor sequences were removed by the fastx clipper tool (http://hannonlab.cshl.edu/fastx_toolkit). Then, all reads were clustered into one tag and those tags shorter than 18 nucleotides were filtered using an in-house built pipeline [57]. Finally, the mirDeep2 program [58] was used for miRNA predication. With mirDeep2, clean tags were first aligned to the sheep genome (NCBI, Oarv3.1) using mappper.pl script without any nucleotide changes (genome hits $<5$ ); a small RNA tag was defined as a miRNA (score $>0$ ) through evaluation of both expression level and biogenesis criteria using miRDeep2.pl scripts. All the mapped tags were annotated by comparison with the ncRNAs (rRNAs, tRNAs, snRNAs, and snoRNA) deposited in the NCBI GenBank database and the Rfam10.1 database using the BLAST program.

All predicated miRNAs were classified into novel (which were not present in MiRbase), known (known ovine miRNAs in MiRbase) and conserved (known miRNAs of other species in MiRbase but have not been found in sheep) miRNAs based on miRbase-v20, and were also simultaneously classified into different miRNAs families based on an established miRNA family system in miRBasev20 using in-house built Perl scripts. The miRNA expression in each library was normalized as FPM values (reads per million), which was calculated as follows: number of sequenced reads/total mapped reads $\times 1,000,000$. The differentially expressed microRNAs among different stages were obtained by the edgeR software package [59] which used an empirical Bayesian method to test differential expression in deep sequencing datasets, and during this process the sequencing depth was normalized using edgeR software. Those miRNAs with $p$-values $<0.001$ were defined as differentially expressed.

\section{Bioinformatic analysis for sequenced mRNAs}

For raw sequenced data (fastq format), quality control and sequence statistics were performed by FastQC version 0.10.1 (http://www.bioinformatics.babraham.ac.uk/projects/ fastqc/). After trimming the low quality bases using inhouse built script, the spliced mapping process was performed by TopHat for each sample against to both genome 
and transcriptome references. Finally, SAMtools (version 0.1.18) and Linux Shell were used to extract mapped reads and other statistical information. Gene expression levels were calculated based on read counts which also included unambiguous mapped reads according to negative binomial distribution. FPKM (Fragments per kilobase of exon per million fragments mapped) values represent expression level of both gene and transcript.

\section{Validation of sequencing data by real-time quantitative PCR}

Validation of sequencing data for five randomly selected miRNAs was performed by real-time quantitative PCR (Q-PCR). The stem-loop primer and Q-PCR pairs are summarized in Additional file 5. The CDNA was synthesized from $1 \mu \mathrm{g}$ of total RNA using a PrimeScript RT reagent Kit (Takara, Dalian, China). Q-PCR was performed using a ABI7500 (Applied Biosystems, CA, USA) and a standardized protocol. In a $20 \mu \mathrm{l}$ reaction mixture, $2.0 \mu \mathrm{l}$ of cDNA (at a 1:4 dilution) was used for amplification, with $10 \mu \mathrm{l}$ of SYBR Premix Ex TaqTM II (TaKaRa), $1.6 \mu \mathrm{l}$ of a miR-specific Primer set $(0.8 \mu \mathrm{l}$ of each forward and reverse primer), $0.4 \mu \mathrm{l}$ ROX Dye II and $6 \mu \mathrm{l}$ of water. The reactions were incubated at $95^{\circ} \mathrm{C}$ for $30 \mathrm{~s}$, followed by 40 cycles of $95^{\circ} \mathrm{C}$ for $5 \mathrm{~s}, 60^{\circ} \mathrm{C}$ for $34 \mathrm{~s}$. The abundance of selected miRNAs was normalized relative to that of U6 snRNA. All reactions were performed in triplicate. The threshold cycle (CT) was determined using the default threshold settings and the data was analyzed using the $2^{-\Delta \Delta \mathrm{Ct}}$ method [60].

\section{miRNA target prediction, analysis of expression} correlation and enriched KEGG pathway annotation

The 3 '-UTR sequences were extracted according to gene annotation information. Potential targets for miRNAs were predicted using the miRanda, RNAhybrid and TargetScan algorithms simultaneously. Parameters were set as follows: score threshold at 50, energy threshold at $-20 \mathrm{kcal} / \mathrm{mol}$, scaling parameter at 4 and gap-open penalty at -2 for miRanda; helix constraint at 2-8, max bulge loop size at 3 , max internal loop size at 3 and energy threshold at -20 $\mathrm{kcal} / \mathrm{mol}$ for RNAhybrid; default parameters were used for TargetScan. The overlapping target genes were selected for further analysis. Correlation between the differentially expressed miRNAs and expression of their target genes was analyzed using $\mathrm{R}$ software. The KEGG pathways were used to perform pathway annotation of miRNA targets. The enrichment $p$ values were calculated using hypergeometric distribution.

\section{piRNA predication}

After removing miRNA tags, tag lengths ranging between 24 to 33 nt were simultaneously aligned to sheep genomic sequences and known piRNAs sequences in piRNABank
[44] using bowtie [61] allowing two mismatches. The mapped reads (genome hits <20) were used to analyze piRNAs. Simultaneously, piRNA sequences and piRNA clusters were also predicted by a de novo method using proTRAC software [62].

\section{Additional files} Additional file 1: Distribution of total small RNA reads and tags by
Solexa sequencing.

Additional file 2: Associations of expressions between the differentially expressed miRNAs and their target genes.

Additional file 3: Gonadal hormone secretion related pathways in which target genes of differentially expressed miRNAs enriched in this study. The pathway maps were downloaded in KEGG database (http://www.genome.jp/kegg/).

Additional file 4: Gonadotropin secretion related pathways in which target genes of differentially expressed miRNAs enriched and their relationship. The pathway map was downloaded in KEGG database (http://www.genome.jp/kegg/).

Additional file 5: Primer sequences for reverse transcription and Q-PCR of five randomly selected miRNAs.

\section{Abbreviations}

sRNAs: Small RNAs; STH: Small tail Han (sheep); FSH: Follicle-stimulating hormone; LH: Luteinizing hormone; TSA: Tan ewes at anestrus in spring; TAL: Tan ewes at luteal phase in autumn; TAP: Tan ewes at proestrus in autumn; TAE: Tan ewes at estrus in autumn; HSL: STH ewes at luteal phase in spring; HSP: STH ewes at proestrus in spring; HSE: STH ewes at estrus in spring; HAE: STH ewes at estrus in autumn.

\section{Competing interests}

The authors declare that they have no competing interests.

\section{Authors' contributions}

$\mathrm{RD}$ and $\mathrm{MXC}$ conceived and designed the experiments. JNH, XJL, JDW and GLC performed sample collection. JXW, ZMY, MS, YG and QM helped carry out the experiments. SHS, DMT, WMZ analyzed the data. RD, SHS and QYL wrote the paper. All authors read and approved the final manuscript.

\section{Acknowledgments}

This work was supported by National Natural Science Foundation of China (Grant No. 31472078), the Earmarked Fund for China Agriculture Research System (Grant No. CARS-39) and Agricultural Science and Technology Innovation Program of China (Grant No. ASTIP-IAS13) to Mingxing Chu, Special Fund for Basic Scientific Research of Institute of Animal Science, Chinese Academy of Agricultural Sciences to Ran Di (Grant No. 2011cj-7) and Qiuyue Liu (Grant No. 2013ywf-zd-1), National Natural Science Foundation of China (Grant No. 31101687) to Ran Di, National Natural Science Foundation of China (Grant No. 31271372) and Beijing Nova Program to Shuhui Song (Grant No. Z121105002512060)

\section{Author details}

${ }^{1}$ Key Laboratory of Farm Animal Genetic Resources and Germplasm Innovation of Ministry of Agriculture, Institute of Animal Science, Chinese Academy of Agricultural Sciences, No. 2, Yuanmingyuan West Rd, Beijing, China. ${ }^{2}$ Core Genomic Facility, Beijing Institute of Genomics, Chinese Academy of Sciences, Beijing, China. ${ }^{3}$ Research Center of Grass and Livestock, Ningxia Academy of Agriculture and Forestry Sciences, Yinchuan, Ningxia, China.

Received: 30 July 2014 Accepted: 8 October 2014 Published: 15 October 2014

\section{References}

1. Tu YR: Sheep and Goat Breeds in China: Shanghai Scientific \& Technology Publishers; 1989. 
2. McBride D, Carre W, Sontakke SD, Hogg CO, Law A, Donadeu FX, Clinton M: Identification of miRNAs associated with the follicular-luteal transition in the ruminant ovary. Reproduct 2012, 144(2):221-233.

3. Ling YH, Ren $\mathrm{CH}$, Guo XF, Xu LN, Huang YF, Luo JC, Zhang YH, Zhang XR, Zhang ZJ: Identification and characterization of microRNAs in the ovaries of multiple and uniparous goats (Capra hircus) during follicular phase. BMC Genomics 2014, 15(1):339.

4. Ji Z, Wang G, Xie Z, Zhang C, Wang J: Identification and characterization of microRNA in the dairy goat (Capra hircus) mammary gland by Solexa deep-sequencing technology. Mol Biol Rep 2012, 39(10):9361-9371.

5. Wu J, Zhu H, Song W, Li M, Liu C, Li N, Tang F, Mu H, Liao M, Li X, Guan W, Li X, Hua J: Identification of conservative microRNAs in Saanen dairy goat testis through deep sequencing. Reprod Domest Anim 2014, 49(1):32-40.

6. Liu Z, Xiao H, Li H, Zhao Y, Lai S, Yu X, Cai T, Du C, Zhang W, Li J: Identification of conserved and novel microRNAs in cashmere goat skin by deep sequencing. PLoS One 2012, 7(12):e50001.

7. Yuan C, Wang X, Geng R, He X, Qu L, Chen Y: Discovery of cashmere goat (Capra hircus) microRNAs in skin and hair follicles by Solexa sequencing. BMC Genomics 2013, 14:511.

8. Zhang XD, Zhang YH, Ling YH, Liu Y, Cao HG, Yin ZJ, Ding JP, Zhang XR: Characterization and differential expression of microRNAs in the ovaries of pregnant and non-pregnant goats (Capra hircus). BMC Genomics 2013, 14:157.

9. Zhang S, Zhao F, Wei C, Sheng X, Ren H, Xu L, Lu J, Liu J, Zhang L, Du L: Identification and characterization of the miRNA transcriptome of Ovis aries. PLoS One 2013, 8(3):e58905

10. Otsuka M, Zheng M, Hayashi M, Lee JD, Yoshino O, Lin S, Han J: Impaired microRNA processing causes corpus luteum insufficiency and infertility in mice. J Clin Invest 2008, 118(5):1944-1954.

11. Yao GD, Yin MM, Lian J, Tian H, Liu L, Li X, Sun F: MicroRNA-224 is involved in transforming growth factor-beta-mediated mouse granulosa cell proliferation and granulosa cell function by targeting Smad4. Mol Endocrinol 2010, 24(3):540-551.

12. Lin F, Li R, Pan ZX, Zhou B, Yu DB, Wang XG, Ma XS, Han J, Shen M, Liu HL miR-26b promotes granulosa cell apoptosis by targeting ATM during follicular atresia in porcine ovary. PLOS One 2012, 7(6):e38640.

13. Grivna ST, Beyret E, Wang Z, Lin H: A novel class of small RNAs in mouse spermatogenic cells. Genes Dev 2006, 20(13):1709-1714.

14. Grivna ST, Pyhtila B, Lin H: MIWI associates with translational machinery and PIWI-interacting RNAs (piRNAs) in regulating spermatogenesis. Proc Natl Acad Sci U S A 2006, 103(36):13415-13420.

15. Lau NC, Seto AG, Kim J, Kuramochi-Miyagawa S, Nakano T, Bartel DP, Kingston RE: Characterization of the piRNA complex from rat testes. Science 2006, 313(5785):363-367.

16. Malone CD, Brennecke J, Dus M, Stark A, McCombie WR, Sachidanandam R, Hannon GJ: Specialized piRNA pathways act in germline and somatic tissues of the drosophila ovary. Cell 2009, 137(3):522-535.

17. Tam OH, Aravin AA, Stein P, Girard A, Murchison EP, Cheloufi S, Hodges E, Anger M, Sachidanandam R, Schultz RM, Hannon GJ: Pseudogene-derived small interfering RNAs regulate gene expression in mouse oocytes. Nature 2008, 453(7194):534-538.

18. Watanabe T, Totoki Y, Toyoda A, Kaneda M, Kuramochi-Miyagawa S, Obata Y, Chiba H, Kohara Y, Kono T, Nakano T, Surani MA, Sakaki Y, Sasaki H: Endogenous siRNAs from naturally formed dsRNAs regulate transcripts in mouse oocytes. Nature 2008, 453(7194):539-543.

19. Yang CX, Du ZQ, Wright EC, Rothschild MF, Prather RS, Ross JW: Small RNA profile of the cumulus-oocyte complex and early embryos in the pig. Biol Reprod 2012, 87(5):117

20. Li CJ, Vagin W, Lee SH, Xu J, Ma SM, Xi HL, Seitz H, Horwich MD, Syrzycka M, Honda BM, Kittler ELW, Zapp ML, Klattenhoff C, Schulz N, Theurkauf WE Weng ZP, Zamore PD: Collapse of germline piRNAs in the absence of argonaute3 reveals somatic piRNAs in flies. Cell 2009, 137(3):509-521.

21. Gerstl MP, Hackl M, Graf AB, Borth N, Grillari J: Prediction of transcribed PIWI-interacting RNAs from CHO RNAseq data. J Biotechnol 2013, 166(1-2):51-57.

22. Ding $X$, Guan $\mathrm{H}$, $\mathrm{Li} \mathrm{H}$ : Characterization of a piRNA binding protein Miwi in mouse oocytes. Theriogenol 2013, 79(4):610-615.

23. Hossain MM, Ghanem N, Hoelker M, Rings F, Phatsara C, Tholen E, Schellander K, Tesfaye D: Identification and characterization of miRNAs expressed in the bovine ovary. BMC Genomics 2009, 10:443.

24. Huang JM, Ju ZH, Li QL, Hou QL, Wang CF, Li JB, Li RL, Wang LL, Sun T, Hang SQ, Gao YD, Hou MH, Zhong JF: Solexa sequencing of novel and differentially expressed microRNAs in testicular and ovarian tissues in Holstein cattle. Int J Biol Sci 2011, 7(7):1016-1026.

25. Tripurani SK, Xiao CD, Salem M, Yao JB: Cloning and analysis of fetal ovary microRNAs in cattle. Anim Reprod Sci 2010, 120(1-4):16-22.

26. Li MZ, Liu YK, Wang T, Guan JQ, Luo ZG, Chen HS, Wang X, Chen L, Ma JD, Mu ZP, Jiang AA, Zhu L, Lang QL, Zhou XC, Wang JY, Zeng WX, Li N, Li K, Gao XL, Li XW: Repertoire of porcine microRNAs in adult ovary and testis by deep sequencing. Int J Bio/ Sci 2011, 7(7):1045-1055.

27. Mishima T, Takizawa T, Luo SS, Ishibashi O, Kawahigashi Y, Mizuguchi Y, Ishikawa T, Mori M, Kanda T, Goto T, Takizawa T: MicroRNA (miRNA) cloning analysis reveals sex differences in miRNA expression profiles between adult mouse testis and ovary. Reproduct 2008, 136(6):811-822

28. Ahn HW, Morin RD, Zhao H, Harris RA, Coarfa C, Chen ZJ, Milosavljevic A Marra MA, Rajkovic A: MicroRNA transcriptome in the newborn mouse ovaries determined by massive parallel sequencing. Mol Hum Reprod 2010, 16(7):463-471.

29. Carletti MZ, Fiedler SD, Christenson LK: MicroRNA 21 blocks apoptosis in mouse periovulatory granulosa cells. Biol Reprod 2010, 83(2):286-295.

30. Zhang JF, Ji XW, Zhou DD, Li YQ, Lin JK, Liu JL, Luo HS, Cui S: miR-143 is critical for the formation of primordial follicles in mice. Front Biosci-Landmark 2013, 18:588-597.

31. Yan GJ, Zhang LX, Fang T, Zhang Q, Wu SG, Jiang Y, Sun HX, Hu YL: MicroRNA-145 suppresses mouse granulosa cell proliferation by targeting activin receptor IB. Febs Lett 2012, 586(19):3263-3270.

32. Jovanovic VP, Sauer CM, Shawber CJ, Gomez R, Wang X, Sauer MV, Kitajewski J, Zimmermann RC: Intraovarian regulation of gonadotropin-dependent folliculogenesis depends on notch receptor signaling pathways not involving delta-like ligand 4 (DII4). Reprod Biol Endocrin 2013, 11:43.

33. Trombly DJ, Woodruff TK, Mayo KE: Suppression of Notch signaling in the neonatal mouse ovary decreases primordial follicle formation. Endocrinol 2009, 150(2):1014-1024.

34. Vorontchikhina MA, Zimmermann RC, Shawber CJ, Tang HY, Kitajewski J: Unique patterns of Notch1, Notch4 and Jagged1 expression in ovarian vessels during folliculogenesis and corpus luteum formation. Gene Expr Patterns 2005, 5(5):701-709.

35. Craig J, Zhu H, Dyce PW, Petrik J, Li J: Leptin enhances oocyte nuclear and cytoplasmic maturation via the mitogen-activated protein kinase pathway. Endocrinol 2004, 145(11):5355-5363.

36. Ferrell JE Jr: Xenopus oocyte maturation: new lessons from a good egg. Bioessays 1999, 21(10):833-842.

37. Carlsbecker A, Lee JY, Roberts CJ, Dettmer J, Lehesranta S, Zhou J, Lindgren O, Moreno-Risueno MA, Vaten A, Thitamadee S, Campilho A, Sebastian J, Bowman $J$, Helariutta Y, Benfey PN: Cell signalling by microRNA165/6 directs gene dose-dependent root cell fate. Nature 2010, 465(7296):316-321.

38. Tian T, Wang $Y$, Wang $H$, Zhu Z, Xiao Z: Visualizing of the cellular uptake and intracellular trafficking of exosomes by live-cell microscopy. J Cell Biochem 2010, 111(2):488-496.

39. Kogure T, Lin WL, Yan IK, Braconi C, Patel T: Intercellular nanovesiclemediated microRNA transfer: a mechanism of environmental modulation of hepatocellular cancer cell growth. Hepatology 2011, 54(4):1237-1248.

40. Mittelbrunn M, Gutierrez-Vazquez C, Villarroya-Beltri C, Gonzalez S, SanchezCabo F, Gonzalez MA, Bernad A, Sanchez-Madrid F: Unidirectional transfer of microRNA-loaded exosomes from T cells to antigen-presenting cells. Nat Commun 2011, 2:282.

41. Aucher A, Rudnicka D, Davis DM: MicroRNAs transfer from human macrophages to hepato-carcinoma cells and inhibit proliferation. J Immunol 2013, 191(12):6250-6260.

42. Karsch FJ, Dahl GE, Evans NP, Manning JM, Mayfield KP, Moenter SM, Foster $\mathrm{DL}$ : Seasonal changes in gonadotropin-releasing hormone secretion in the ewe: alteration in response to the negative feedback action of estradiol. Biol Reprod 1993, 49(6):1377-1383.

43. Barrell GK, Moenter SM, Caraty A, Karsch FJ: Seasonal changes of gonadotropin-releasing hormone secretion in the ewe. Biol Reprod 1992 46(6):1130-1135.

44. Sai Lakshmi S, Agrawal S: piRNABank: a web resource on classified and clustered Piwi-interacting RNAs. NuCl Acid R 2008, 36(Database issue):D173-D177.

45. Houwing S, Kamminga LM, Berezikov E, Cronembold D, Girard A, van den Elst H, Filippov DV, Blaser H, Raz E, Moens CB, Plasterk RH, Hannon GJ, Draper BW, Ketting RF: A role for Piwi and piRNAs in germ cell maintenance and transposon silencing in zebrafish. Cell 2007, 129(1):69-82. 
46. Girard A, Sachidanandam R, Hannon GJ, Carmell MA: A germline-specific class of small RNAs binds mammalian Piwi proteins. Nature 2006, 442(7099):199-202

47. Lim SL, Tsend-Ayush E, Kortschak RD, Jacob R, Ricciardelli C, Oehler MK, Grutzner F: Conservation and expression of PIWI-Interacting RNA pathway genes in male and female adult gonad of amniotes. Biol Reprod 2013, 89(6):136.

48. Minakhina S, Changela N, Steward R: Zfrp8/PDCD2 is required in ovarian stem cells and interacts with the piRNA pathway machinery. Development 2014, 141(2):259-268.

49. Yan Z, Hu HY, Jiang X, Maierhofer V, Neb E, He L, Hu YH, Hu H, Li N, Chen W, Khaitovich P: Widespread expression of piRNA-like molecules in somatic tissues. Nucl Acid R 2011, 39(15):6596-6607.

50. Liu G, Lei B, Li Y, Tong K, Ding Y, Luo L, Xia X, Jiang S, Deng C, Xiong Y, Li F: Discovery of potential piRNAs from next generation sequences of the sexually mature porcine testes. PLoS One 2012, 7(4):e34770.

51. Campbell BK, Gordon BM, Scaramuzzi RJ: The effect of ovarian arterial infusion of transforming growth factor alpha on ovarian follicle populations and ovarian hormone secretion in ewes with an autotransplanted ovary. J Endocrinol 1994, 143(1):13-24.

52. McNeilly JR, McNeilly AS, Walton JS, Cunningham FJ: Development and application of a heterologous radioimmunoassay for ovine follicle-stimulating hormone. J Endocrinol 1976, 70(1):69-79.

53. McNeilly AS, Jonassen JA, Fraser HM: Suppression of follicular development after chronic LHRH immunoneutralization in the ewe. J Reprod Fertil 1986, 76(1):481-490.

54. Djahanbakhch O, Swanton IA, Corrie JE, McNeilly AS: Prediction of ovulation by progesterone. Lancet 1981, 2(8256):1164-1165.

55. McNeilly AS: Changes in FSH and the pulsatile secretion of LH during the delay in oestrus induced by treatment of ewes with bovine follicular fluid. J Reprod Fertil 1984, 72(1):165-172.

56. Cumming IA, Brown JM, Goding JR, Bryant GD, Greenwood FC: Secretion of prolactin and luteinizing hormone at oestrus in the ewe. $J$ Endocrinol 1972, 54(2):207-213.

57. Zhao WM, Liu WF, Tian DM, Tang BX, Wang YQ, Yu CX, Li RJ, Ling YC, Wu JY, Song SH, Hu SN: wapRNA: a web-based application for the processing of RNA sequences. Bioinformatics 2011, 27(21):3076-3077.

58. Friedlander MR, Mackowiak SD, Li N, Chen W, Rajewsky N: miRDeep2 accurately identifies known and hundreds of novel microRNA genes in seven animal clades. Nucl Acid R 2012, 40(1):37-52.

59. Robinson MD, McCarthy DJ, Smyth GK: edgeR: a bioconductor package for differential expression analysis of digital gene expression data. Bioinformatics 2010, 26(1):139-140.

60. Livak KJ, Schmittgen TD: Analysis of relative gene expression data using real-time quantitative PCR and the 2(T)(-Delta Delta C) method. Methods 2001, 25(4):402-408.

61. Langmead B, Trapnell C, Pop M, Salzberg SL: Ultrafast and memory-efficient alignment of short DNA sequences to the human genome. Genome Biol 2009, 10(3):R25

62. Rosenkranz D, Zischler H: proTRAC - a software for probabilistic piRNA cluster detection, visualization and analysis. BMC Bioinformatics 2012, 13:5.

\section{Submit your next manuscript to BioMed Central and take full advantage of:}

- Convenient online submission

- Thorough peer review

- No space constraints or color figure charges

- Immediate publication on acceptance

- Inclusion in PubMed, CAS, Scopus and Google Scholar

- Research which is freely available for redistribution

Submit your manuscript at www.biomedcentral.com/submit 